\title{
REFRESMENT Diagnostic difficulty in bipolar disorder
}

\author{
Kate E. A. Saunders
}

Kate Saunders is a clinical researcher and a consultant psychiatrist in the University of Oxford's Department of Psychiatry at Warneford Hospital, Oxford. She is also the course organiser for the Oxford MRCPsych Course. Her primary research interests are in mood instability, borderline personality disorder and bipolar disorder.

Correspondence Dr Kate E. A. Saunders, University Department of Psychiatry, Warneford Hospital, Oxford OX3 7JX, UK. Email: kate. saunders@psych.ox.ac.uk

\section{Copyright and usage}

(C) The Royal College of Psychiatrists 2017.

\begin{abstract}
SUMMARY
Bipolar disorder is a common mental disorder that can be challenging to diagnose. This brief article outlines how bipolar disorder is diagnosed and how it can be distinguished from other disorders with similar phenotypes.
\end{abstract}

\section{DECLARATION OF INTEREST}

None

Bipolar disorder is a relapsing and remitting condition that affects around $2 \%$ of the population. Current diagnostic classifications define it as a disorder comprising discrete episodes of mania or depression interspersed by periods of stability sometimes referred to as euthymia. The diagnosis of bipolar disorder is based wholly on clinical history and current mood state, and is contingent on the presence of at least one episode of mania or hypomania. The diagnostic criteria for mania were updated in DSM-5 (American Psychiatric Association 2013) and now require that any mood change (elation or irritability) is accompanied by persistently increased activity or energy levels (Box 1). Prospective mood monitoring reveals a more accurate, but more complex phenotype where mood instability predominates.

The changeable nature of bipolar disorder, as well as the limitations of current diagnostic systems, mean that it can be challenging to diagnose or distinguish from other disorders (Phillips 2013). Concerns have been raised regarding expanding prevalence rates for bipolar disorder. Significant increases in the diagnosis of the disorder in paediatric populations have been observed, but rates in adults have remained largely unchanged and there is a remarkable degree of consistency in the epidemiology of bipolar disorder across countries despite diverse study designs.

\section{Bipolar or unipolar depression?}

Many people experience lengthy delays in receiving a diagnosis of bipolar disorder because their initial mood episodes are depressive in nature. Approximately $20 \%$ of all those referred to secondary care with a depressive illness develop bipolar disorder.
At present there is no agreed method for distinguishing unipolar from bipolar depression, but factors such as a family history of bipolar disorder and the presence of 'atypical depressive features' (e.g. hypersomnia and weight gain) may be suggestive (Forty 2008). In those in whom the risk of bipolarity is high, clinicians should be judicious in their use of antidepressant medications and monitor carefully for the emergence of manic or mixed features. Rating scales such as the Mood Disorder Questionnaire (MDQ) may be helpful in identifying possible bipolarity, and clinicians should routinely enquire about mood elevation in all patients presenting with depression (Kendall 2014).

\section{Bipolar disorder or borderline personality disorder?}

Another common differential diagnosis is borderline personality disorder. There is significant overlap between the diagnostic criteria of the two disorders and about $15 \%$ of patients with bipolar disorder will have comorbid borderline

BOX 1 Summary of DSM-5 criteria for a manic episode

A defined period of elated or irritable mood and increased activity or energy lasting at least a week (at least 4 days for hypomania) associated with three (four if mood is irritable) or more of the following:

- racing thoughts or flight of ideas

- more talkative

- easily distracted

- excessive involvement in activities

- engaging in risky behaviours

- grandiosity or increased sense of own abilities

- less need for sleep

- increase in goal-directed activity

and also associated with a significant impairment in function or with psychosis.

For hypomania, change in functioning must be significant enough to be observed by others while not being significant enough to cause marked impairment or necessitate hospital admission.

(American Psychiatric Association 2013) 
personality disorder. Some authors have proposed that borderline personality disorder is in fact part of the bipolar spectrum, but a growing body of evidence suggests that the two are distinct and can be objectively distinguished using cognitive tasks. Clinically, the most consistent predictors of borderline personality disorder are childhood sexual abuse, childhood depersonalisation, personality variables relating to relationship difficulties, sensitivity to criticism and the absence of any family history of bipolar disorder. The pervasive nature of the symptoms is important to establish in confirming the presence of borderline personality disorder. However, all of these features need to be considered in the context of the clinical presentation (Saunders 2015).

\section{Bipolar disorder or ADHD}

In children (and increasingly in adults), attentiondeficit hyperactivity disorder (ADHD) is a common differential diagnosis. ADHD may be overrepresented in bipolar disorder, although the reason for this is unclear. The classic symptoms of mania such as grandiosity, hypersexuality and racing thoughts are not found in ADHD alone. Symptoms of ADHD (hyperactivity, impulsivity, inattention) must be present in a minimum of two settings and emerge before the age of 7. Bipolar disorder is rare in this age group. The courses of the disorders differ significantly too. Bipolar disorder has an episodic course (albeit with significant subsyndromal symptoms), whereas the symptoms of ADHD are more continuous in nature and occur independently of mood state (Gleason 2012).

\section{Other differential diagnoses}

Manic states due to general medical conditions are often clinically indistinguishable from bipolar mania, but there are likely to be signs and symptoms of underlying organic illness, for example hyperthyroidism. Prescribed (e.g. steroids) and illicit substances can also mimic manic states. Symptoms should resolve on the withdrawal of the substance, although they can persist for a number of weeks.

Bipolar disorder can be particularly difficult to identify in people with comorbid psychiatric diagnoses and/or substance misuse. However, careful attention to the clinical history and diagnostic criteria is imperative, and prospective mood monitoring can be helpful where uncertainty remains.

\section{References}

American Psychiatric Association (2013) Diagnostic and Statistical Manual of Mental Disorders (5th edn) (DSM-5). APA.

Forty L, Smith D, Jones L, et al (2008) Clinical differences between bipolar and unipolar depression. British Journal of Psychiatry, 192: 388-9.

Gleason A, Castle D (2012) Adult attention-deficit hyperactivity disorder and bipolar disorder. Advances in Psychiatric Treatment, 18: 198-204.

Kendall T, Morriss R, Mayo-Wilson E, et al (2014) Assessment and management of bipolar disorder: summary of updated NICE guidance. BMJ, 349: 5673.

Phillips ML, Kupfer DJ (2013) Bipolar disorder diagnosis: challenges and future directions. Lancet, 381: 1663-71.

Saunders KEA, Bilderbeck AC, Price JR, et al (2015) Distinguishing bipolar disorder from borderline personality disorder: a study of current clinical practice. European Psychiatry, 30: 965-74. 